\title{
Clinical importance of eflornithine ( $\alpha$-difluoromethylornithine) for the treatment of malignant gliomas
}

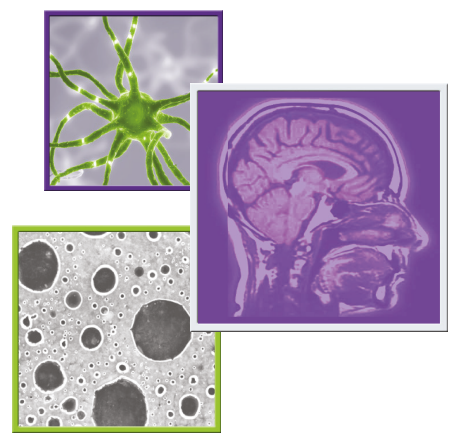

\author{
Victor A Levin*,1, Sandra E Ictech ${ }^{1}$ \& Kenneth R Hess ${ }^{2}$ \\ ${ }^{1}$ Department of Neuro-Oncology, The University of Texas MD Anderson Cancer Center, Houston, TX 77030-4009, USA \\ ${ }^{2}$ Department of Biostatistics, The University of Texas MD Anderson Cancer Center, Houston, TX 77030-4009, USA \\ *Author for correspondence: vlevin11@gmail.com
}

\section{Practice points}

- Eflornithine ( $\alpha$-difluoromethylornithine) is a specific and irreversible inhibitor of ornithine decarboxylase with activity against glial tumors.

- Eflornithine has good bioavailability and can be given three-times daily for 14 days with 7-14-day rest periods between cycles.

- Eflornithine has activity against recurrent gliomas as a single agent.

- Eflornithine in combination with procarbazine, lomustine, vincristine (PCV) chemotherapy is active against anaplastic gliomas with improvement in progression-free survival and overall survival of about 2.5 years over PCV therapy.

- When eflornithine was added to PCV, the most frequent grade 2 toxicities were diarrhea, anemia and thrombocytopenia; the most frequent grade 3 toxicities were diarrhea, anemia and nausea.

- With more intense dosing of eflornithine in single-agent studies, the most frequent grade 2 toxicities were diarrhea, granulocytopenia and anemia; the most frequent grade 3 toxicities were hearing impairment, tinnitus and granulocytopenia.

This review covers the literature between 1989 and 2007 on studies relevant to the neuro-oncology usage of eflornithine ( $\alpha$-difluoromethylornithine), an oral agent that irreversibly inhibits the enzyme ornithine decarboxylase. It covers the use of eflornithine, alone or in combination, to treat high-grade gliomas. In addition, we provide an update on overall survival from The University of Texas MD Anderson Cancer Center Community Clinical Oncology Program and Clinical Trials Data Office that demonstrates a meaningful benefit in overall survival for eflornithine as a single agent and in combination with nitrosourea-based therapies for anaplastic gliomas. We also provide a framework for understanding the basis and study design of the ongoing pivotal, registrational Phase III multicenter trial for recurrent/progressive anaplastic astrocytoma.

First draft submitted: 14 September 2017; Accepted for publication: 14 December 2017; Published online: 30 January 2018

Keywords: astrocytoma • anaplastic oligoastrocytoma • anaplastic oligodendroglioma • glioblastoma • polyamine inhibition

Eflornithine, ( $\alpha$-difluoromethylornithine, DFMO) is an irreversible and specific inhibitor of ornithine decarboxylase, one of the rate-limiting enzymes in the synthesis of polyamines and a recognized target in cancer research. Since inhibition of ornithine decarboxylase is known to lead to cellular effects that influence tumor cell growth [1,2], eflornithine has been evaluated as a treatment for various cancers in multiple clinical studies (reviewed by Bachmann and Levin [2]). In these studies, eflornithine was found to be active against some leukemias and solid tumors, such as breast, colon, cervical, small-cell lung cancer and melanoma.

Eflornithine is not metabolized to a significant extent, is excreted in the urine unchanged and has approximately $80 \%$ oral bioavailability [3,4]. It can be taken orally in gram quantities multiple times daily for periods of weeks with modest side effects, including gastrointestinal (GI) toxicity, such as loose stools and diarrhea, primarily because of its osmotic load on the GI tract. Another observed adverse event is a decline in sensorineural hearing in a

Future $\because$ Medicine 
low percentage of patients, which is usually reversible once eflornithine is stopped. When used with cytotoxic chemotherapy agents, eflornithine may modestly augment myelotoxicity [3,5-9]. Oral eflornithine has a nominal biological plasma half-life of $6-12 \mathrm{~h}$ when chronically dosed [10,11], and has historically been given chronically on either four-times a day or three-times a day schedules in cancer patients.

Eflornithine has been under extensive investigation since the 1980s for various indications. It is approved for marketing in the USA and Europe for topical use as a hair growth retardant (Vaniqa ${ }^{\circledR}$ eflorinthine cream, Allergan, CA, USA). It has been previously developed and authorized as an antiprotozoan treatment for trypanosomiasis and is currently used in those areas of the world affected by this disease.

More than 390 patients with high-grade gliomas, including anaplastic astrocytoma (AA) have been treated with eflornithine in clinical trials between 1989 and 2007. The published studies have evaluated various methods of administration and dose regimens, and have included eflornithine use as a single agent, as well as in combination with other therapies, such as mitoguazone (MGBG), BCNU (1,3-bis(2-chloroethyl)-1-nitrosourea) and PCV (procarbazine, lomustine, vincristine).

This paper summarizes the results of all published studies of eflornithine for the treatment of gliomas, and provides most recent updates for the Phase II single-agent eflornithine study [9] and the randomized Phase III studies of eflornithine-PCV versus PCV [7,8], which lead to the design of the current ongoing clinical trial in recurrent AA (STELLAR trial) [12].

\section{Studies of eflornithine alone \& in combination with mitoguazone}

A total of 166 patients, with 134 evaluable, have been studied in two clinical trials of eflornithine in combination with MGBG [5,9]. The first clinical trial was a two-arm, 45-patient study that compared different schedules of dosing of eflornithine in combination with MGBG and the second trial was a 121-patient study that evaluated eflornithine as a single agent versus an MGBG-eflornithine combination.

In the initial eflornithine-MGBG Phase I/II publication, two dose schedules combining eflornithine with MGBG were used (see Table 1). Initial dosing in group I patients led to significant toxicity in 12 patients that was similar in severity to that observed by another group [13], leading to a better tolerated group II schedule (Table 1). Of the 45 patients with recurrent CNS tumors treated in the study, 33 were evaluable for response; of these, eight patients from Group I and 19 patients from Group II had an infiltrative cerebral glioma that could be evaluated for response. Combining the two groups, there were seven astrocytoma, $13 \mathrm{AA}$ and anaplastic oligoastrocytoma (AOA), six glioblastoma (GBM) and one brainstem glioma.

Efficacy was measured by radiographic response and time to tumor progression (TTP) from initiation of eflornithine therapy. Responses were observed in 4/19 infiltrative glioma patients in group II. Median TTP was 16 weeks in the $11 \mathrm{AA}$ and $\mathrm{AOA}(\mathrm{AA} / \mathrm{AOA})$ patients and 51 weeks in the 6 astrocytoma patients (Table 1). Group II dose-limiting adverse events were GI, myelotoxicity and tinnitus. Hearing loss was not significant, and did not result in dose modification in any of the patients.

It is important to note that the total daily exposure to eflornithine was $4 \mathrm{~g} / \mathrm{m}^{2}$ over a maximum dosing period of 56 days for a total of $168 \mathrm{~g} / \mathrm{m}^{2}$. Improved efficacy was seen in following studies as the daily eflornithine dose was increased.

The results and insights from the prior study led to an Orphan Drug Grant-sponsored randomized Phase II study to evaluate single-agent eflornithine versus a combination of eflornithine and MGBG for the treatment of recurrent malignant gliomas. This was study T88-0236 (CTEP) and DM88-130 (The University of Texas MD Anderson Cancer Center [MDACC] [9]. Patients were randomized to receive either eflornithine as sole therapy or a combination of eflornithine with MGBG. In both cases, treatment cycles were repeated every 63 days (see Table 1 for details). Efficacy response was measured by TTP from initiation of eflornithine therapy.

Due to unanticipated fulminant and fatal hepatotoxicity observed in two study patients (ages 32 and 35) and two other patients participating in an unpublished prostate cancer trial, the US FDA requested that enrollment in the eflornithine-MGBG combination arm of the study be halted with accrual terminated at 23 patients (21 evaluable).

The single agent eflornithine arm continued to accrue and enrolled a total of 98 patients before the study was closed. From the group of 98 patients there were 80 patients evaluable for response and 86 evaluable for toxicity. In this study, grade 3 and 4 toxicities for eflornithine consisted mainly of GI-related complaints (nausea, vomiting, diarrhea), hearing loss and hematologic abnormalities (thrombocytopenia, leukopenia, granulocytopenia). 
Table 1. Results of Phase I/II studies of eflornithine with mitoguazone, Phase II study of eflornithine with carmustine and Phase II study of eflornithine with $\alpha$-IFN for infiltrative malignant gliomas.

\begin{tabular}{|c|c|c|c|c|}
\hline Study & $\mathrm{N}$ & Drug schedule & Efficacy & Safety \\
\hline $\begin{array}{l}\text { Phase I study of } \\
\text { eflornithine + MGBG }\end{array}$ & 45 & $\begin{array}{l}\text { Group I: eflornithine on days } 1-42 \text { at } \\
1 \mathrm{~g} / \mathrm{m}^{2}, \mathrm{q} .6 \mathrm{~h} \text { orally and MGBG on days } \\
14,28 \text { and } 42 \text { at } 350 \mathrm{mg} / \mathrm{m}^{2} \text { iv. } \\
\text { Group II: eflornithine on days } 1-14 \text {, } \\
21-35 \text { and } 42-56 \text { at } 1.33 \mathrm{~g} / \mathrm{m}^{2}, \mathrm{q} .8 \mathrm{~h} \\
\text { orally, and MGBG on days } 14,35 \text { and } 56 \\
\text { at } 200 \mathrm{mg} / \mathrm{m}^{2} \text { iv. }\end{array}$ & $\begin{array}{l}\text { Of } 21 \text { group II patients, the mTTP for six } \\
\text { astrocytoma (three responses and three } \\
\text { SDs) was } 51 \text { weeks and for } 11 \text { AA/AOA } \\
\text { (one response and six SDs) was } 16 \text { weeks }\end{array}$ & $\begin{array}{l}\text { Gastrointestinal toxicity, myelotoxicity } \\
\text { and tinnitus were dose limiting in this } \\
\text { study. Hearing loss was not significant } \\
\text { and did not result in dose modification } \\
\text { in any of the patients }\end{array}$ \\
\hline $\begin{array}{l}\text { Phase II randomized } \\
\text { study of } \\
\text { eflornithine + MGBG vs } \\
\text { eflornithine }\end{array}$ & 121 & $\begin{array}{l}\text { Eflornithine: } 3.6 \mathrm{~g} / \mathrm{m}^{2} \mathrm{q} .8 \mathrm{~h} \text { on days } \\
1-14,22-35 \text { and } 43-56 \text {. } \\
\text { Eflornithine with MGBG: eflornithine } \\
1.8 \mathrm{~g} / \mathrm{m}^{2} \mathrm{q} .8 \mathrm{~h} \text { on days } 1-14,22-35 \text { and } \\
43-56 \text { plus MGBG, } 200 \mathrm{mg} / \mathrm{m}^{2} \text { iv. on } \\
\text { days } 14,35 \text { and } 56 ; \text { cycles were repeated } \\
\text { every } 63 \text { days }\end{array}$ & $\begin{array}{l}\text { For single-agent arm: } \\
45 \% \text { of } 44 \text { AG patients had some } \\
\text { benefit from eflornithine with four PRs, } \\
\text { nine MRs and seven with SD. Median } \\
\text { time free of progression for this group } \\
\text { of } 20 \text { patients was } 49 \text { weeks. } \\
\text { For GBM patients, } 18 \% \text { benefited from } \\
\text { eflornithine with two PR, two MR and } \\
\text { two SD responses }\end{array}$ & $\begin{array}{l}\text { DFMO + MGBG are terminated after } 23 \\
\text { patients due to fatal hepatic toxicity in } \\
\text { two patients. } \\
\text { Single agent: toxicities were reported } \\
\text { for } 86 \text { of the patients treated with } \\
\text { eflornithine. Grade } 3 \text { and } 4 \text { toxicities in } \\
\text { the eflornithine-only arm consisted } \\
\text { mainly of gastrointestinal-related } \\
\text { complaints (nausea, vomiting, diarrhea), } \\
\text { hearing loss and hematologic } \\
\text { abnormalities (thrombocytopenia, } \\
\text { leukopenia, granulocytopenia) }\end{array}$ \\
\hline $\begin{array}{l}\text { Phase II } \\
\text { eflornithine + BCNU }\end{array}$ & 38 & $\begin{array}{l}\text { Eflornithine, } 2.0 \mathrm{~g} / \mathrm{m}^{2} \text { orally every } 8 \mathrm{~h} \\
\text { on days } 1-14 \text { and } 29-42 \text {, and BCNU, } \\
210 \mathrm{mg} / \mathrm{m}^{2} \text { on day } 15 ; \text { treatment was } \\
\text { repeated every } 8 \text { weeks for six courses } \\
\text { or until disease progression }\end{array}$ & $\begin{array}{l}\text { AA/AOA: PR observed by enhanced CT } \\
\text { scan in } 10 \%(2 / 21) \text { and SD in } 48 \% \\
(10 / 21) \text { of patients. mOS for the } \\
\text { AA/AOA group of } 21 \text { was } 56 \text { weeks. Of } \\
\text { interest was that in the combined } \\
\text { PR + SD AA/AOA group of } 12 \text { patients, } \\
\text { mOS was } 119 \text { weeks. } \\
\text { GBM: minimal activity in } 12 \text { patients } \\
\text { with a median time to tumor } \\
\text { progression of } 8 \text { weeks and mOS of } \\
21 \text { weeks. } \\
\text { Brainstem glioma: of five patients with } \\
\text { brainstem tumors, three were alive with } \\
\text { SD at } 77,93 \text { and } 220 \text { weeks }\end{array}$ & $\begin{array}{l}\text { Moderate toxicity was observed at } \\
\text { these doses for myelosuppression } \\
\text { (except for one patient who died of } \\
\text { sepsis due to severe myelosuppression), } \\
\text { dyspnea and tinnitus. One patient had } \\
\text { bilateral sensorineural hearing loss prior } \\
\text { to the start of therapy and progressive } \\
\text { hearing loss on the side of the } \\
\text { brainstem tumor; audiogram performed } \\
6 \text { months after the cessation of } \\
\text { chemotherapy continued to show } \\
\text { progressive unilateral hearing loss }\end{array}$ \\
\hline $\begin{array}{l}\text { Phase II } \\
\text { eflornithine }+\alpha-\text { IFN }\end{array}$ & 29 & $\begin{array}{l}\text { Eflornithine, } 2.25 \mathrm{~g} / \mathrm{m}^{2} \mathrm{q} 6 \mathrm{~h} \text { on days } 1-7 \\
\text { and } \alpha-\mathrm{IFN} 36 \mathrm{MU} / \mathrm{m}^{2} \text { subcutaneously on } \\
\text { days } 3-7\end{array}$ & $\begin{array}{l}\text { No responses in } 29 \text { evaluable patients, } \\
\text { although SD that exceeded } 6 \text { months in } \\
24 \%(7 / 29) \text { of patients and was } \\
\text { considered due to eflornithine } \\
\text { administration }\end{array}$ & $\begin{array}{l}\text { Grade } 3 \text { and } 4 \text { toxicities were mainly } \\
\text { gastrointestinal (nausea, vomiting, } \\
\text { diarrhea), hematologic (leukopenia), } \\
\text { myalgia, fever, chills and lethargy }\end{array}$ \\
\hline
\end{tabular}

AA: Anaplastic astrocytoma; AG: Anaplastic glioma; AOA: Anaplastic oligoastrocytoma; BCNU: Carmustine 1,3-bis(2-chloroethyl)-1-nitrosourea; DFMO: $\alpha$-difluoromethylornithine; GBM: Glioblastoma; iv.: Intravenously; MGBG: Methylglyoxal-bis(guanylhydrazone); mOS: Median overall survival; MR: Minor response; mTTP: Median time to tumor progression; PFS: Progression-free survival; PR: Partial response; q.: Every; SD: Stable disease.

The radiographic response rate, measured by contrast-enhanced computed tomography (CT) or MRI used earlier criteria adapted for CT and MRI [9,14]. It was shown that $45 \%$ of 44 anaplastic glioma (AG) patients had some benefit from eflornithine with four partial responses (PR), nine minor responses (MR) and seven with stable disease (SD). Since this study enrolled all patients regardless of the amount of prior therapy they had received, nine patients progressed before taking one course of therapy, implying that the tumors of this subset of AG patients were now growing as GBM by the time they started eflornithine therapy, either because of pre-existing molecular features or phenotypic transformation [15]. It was found that the median time free of progression for the subgroup of 20 patients who had a PR, MR or SD was 49 weeks. Of 36 evaluable GBM patients, only $18 \%$ benefited from eflornithine with two PR, two MR and two SD responses. This subgroup of six patients had a median time free of progression of 32 weeks [9].

In 2001, with the help of MDACC Clinical Trials Data Office, the overall survival (OS) for patients who received eflornithine as a single agent in this study was analyzed. In addition, pathology was reviewed to determine the outcomes of those recurrent AG patients with the diagnosis of recurrent AA. For the 34 patients with recurrent AA, median progression-free survival (mPFS) was 17.6 weeks with median OS (mOS) being 100 weeks or 23 months (Figure 1A).

These 2001 data suggest that single-agent eflornithine produced a 23-month mOS result, which is better than the mOS reported by Wong et al. from consecutive Phase II trials of AA patients studied at MDACC [16]. In the Wong study, they computed an mOS of 10.8 months for 150 Phase II study patients with recurrent AA (rAA). It 
(A)

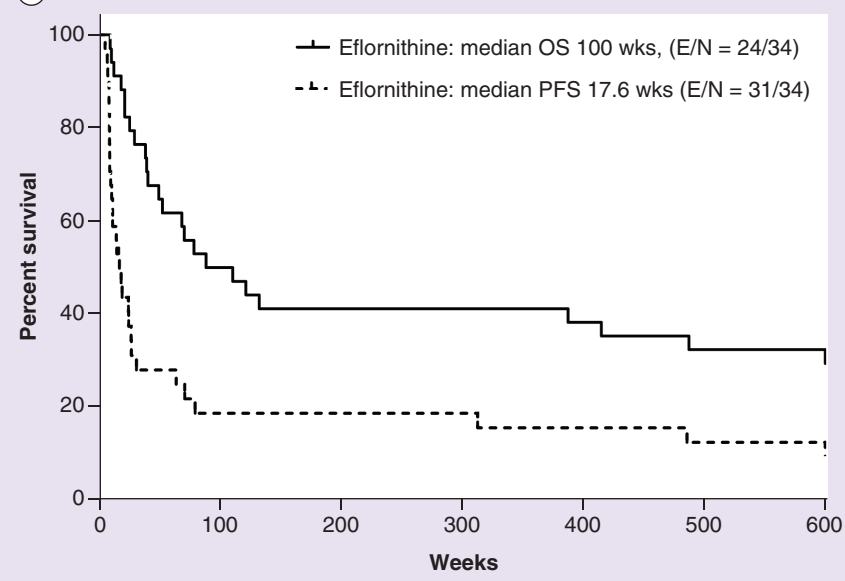

(B)

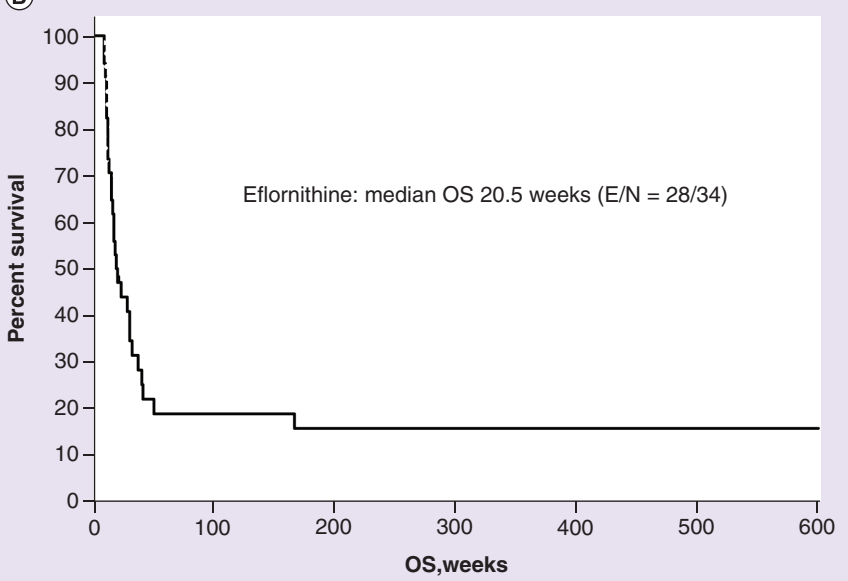

Figure 1. Kaplan-Meier representations of progression-free survival and overall survival of eflornithine patients treated on US FDA Orphan Drug Grant Study T88-0236 and study DM88-130 with diagnosis of AA and GBM. PFS and OS for (A) AA and overall survival for (B) GBM were updated in 2001. There were 34 evaluable AA from the original study and 38 GBM [9]. AA: Anaplastic astrocytoma; GBM: glioblastoma; PFS: Progression-free survival; OS: Overall survival.

should be noted that these rAA patients included the patients studied in the eflornithine single-arm study described above. When the Wong data are re-analyzed after removing the 54 patients treated with eflornithine, the mOS for the remaining patients dropped to 8.7 months with OS at 2 years of $25 \%$.

Further, this single-agent eflornithine data appear encouraging compared with the Chamberlain $e t$ al. recent evaluation of lomustine in rAA patients who had progressed after radiation therapy (RT) and temozolomide. In a retrospective study of $35 \mathrm{rAA}$ patients, they found an mOS of 10 months with no OS at 2 years [17]. In our study, eflornithine demonstrated an mOS of 23 months and OS at 2 years of $48 \%$, and suggests that eflornithine as a single agent may lead to better outcomes for rAA patients compared with lomustine or the many Phase II therapies assessed at MDACC and cited earlier. Both studies used criteria of radiographically measurable recurrent tumor and determination of initial AA histology by microscopy. In addition, Chamberlain obtained molecular studies, not available at the time of our clinical trial, that showed no patients had chromosome $1 \mathrm{p} / 19 \mathrm{q}$ co-deletion, $86 \%$ had IDHI mutation by immunohistochemistry (IHC), $28 \%$ with $A T R X$ mutation by IHC and $17 \%$ with $M G M T$ promoter methylation. Thus, about $14 \%$ of his patients had a genotype more consistent to GBM than AA.

In addition to the much improved mOS for $\mathrm{AA}$ and $\mathrm{AOA}$ over published reports, the OS for these patients with confirmed recurrent AA diagnosis at 2 years was $48 \%$. It may be that this OS result was due to the increased total daily dose of eflornithine at $10.8 \mathrm{~g} / \mathrm{m}^{2}$, which was much higher than used in the prior study, and increased dose duration of up to 2 years. On the other hand, survival following tumor progression/recurrence, especially for WHO grade 3 gliomas, can be confounded by the impact of therapies used prior to randomization as well as those used at progression.

For the $34 \mathrm{AA}$, there were seven patients who failed only RT before starting study. Of the remaining 27 patients, 19 started eflornithine after failing RT and one chemotherapy, while eight patients started eflornithine after RT and at least two prior chemotherapies. All the 27 patients having chemotherapy before eflornithine received at least one nitrosourea, 15/27 also received procarbazine, 6/27 also received dibromodulcitol or a platinum compound, and all but one also received one to three additional anticancer agents in various combinations. Thus, one would presume that the seven that failed RT before receiving eflornithine would be candidates for cytotoxic drugs after progression and might do better than those who were treated with chemotherapy prior to eflornithine. Interestingly, the seven patients who failed RT had an mPFS of 9.3 weeks and mOS of 89 weeks. If these seven patients were then removed from the combined AA/AOA group of 38 cases or the AA group of 34 cases, the mPFS was 18.9 weeks and mOS 122 weeks in both cases (Figure 2).

These outcomes are interesting from a couple of perspectives. First, it shows that those seven people who failed only RT did not appreciably impact mPFS or reduce mOS of the whole study; this was true whether considering $\mathrm{AA}$ or a combined AA/AOA cohort. In addition, given the number of cytotoxic agents' patients took before starting 


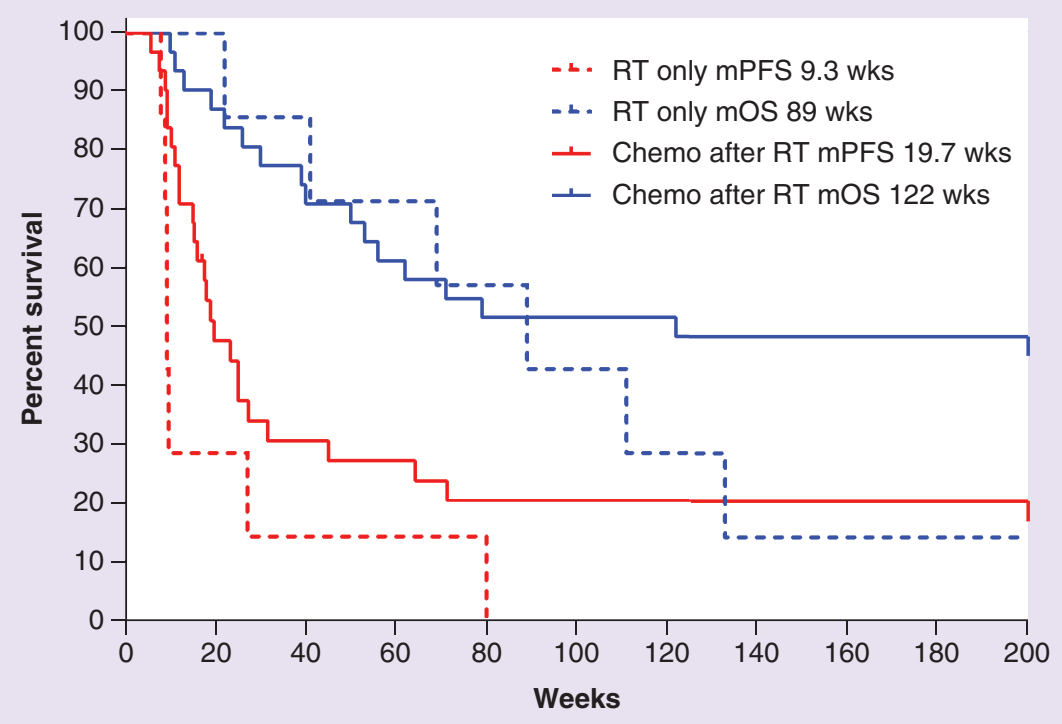

Figure 2. Study DM88-130 subgroup of patients failing RT compared with those who failed RT and adjuvant chemotherapy prior to eflornithine. The median values were the same whether we used 34 AA or 38 AA/anaplastic oligoastrocytoma as only 7 AA received RT only prior to eflornithine.

AA: Anaplastic astrocytoma.

the eflornithine study, it is unlikely, and probably impossible, for the patients who received chemotherapy after RT to have received effective secondary chemotherapy when they failed eflornithine as none would have been available to them in 1991 when this study ended. Unanswered, however, is the explanation why a study with an mPFS of approximately 19 weeks produces an mOS of 122 weeks? As eflornithine is more of a cytostatic drug than a cytotoxic drug, it may be that the lasting effect of eflornithine on the tumor may be its ability to reduce future mutations and, thereby, transformation to a more malignant phenotype. Further study is needed to understand this important observation.

\section{Study of eflornithine in combination with BCNU}

Based on growing experimental evidence from culture and rodent models that BCNU, when dosed following eflornithine, led to more cell kill and antitumor activity than either agent alone [18-21], we conducted a short Phase I/II clinical trial of a BCNU-eflornithine combination in patients with recurrent malignant glioma [6]. The study was designed to evaluate the toxicity of the combination and determine the strength of the signal for antitumor activity of the combination for a future randomized Phase III study. In the study (details in Table 1), 38 adults and children with primary recurrent gliomas and GBM were treated with eflornithine, $2 \mathrm{~g} / \mathrm{m}^{2}$ orally every $8 \mathrm{~h}$ on days 1-14 and 29-42, and intravenously (iv.) carmustine, $210 \mathrm{mg} / \mathrm{m}^{2}$ on day 15 . The treatment was repeated every 8 weeks for six courses or until the disease progressed. Only moderate toxicity was observed at these doses for myelosuppression (except for one patient who died of sepsis due to severe myelosuppression), dyspnea and tinnitus. One patient who had bilateral sensorineural hearing loss prior to the start of therapy had progressive hearing loss on the side of the brain stem tumor. An audiogram performed 6 months after the cessation of chemotherapy continued to show progressive unilateral hearing loss. It was not certain if this loss was attributable to the chemotherapy or prior tumor irradiation. In this trial, TTP was measured from initiation of therapy to radiographic progression and objective response rates were also determined [6].

Determination of response was possible in 21 patients with the diagnosis of AA or AOA. Of these patients, all 21 received prior RT and 19\% (4/21) also received an alkylating agent as adjuvant chemotherapy. In this patient population, a PR observed by enhanced CT scan was found in $10 \%(2 / 21)$ and SD in $48 \%(10 / 21)$. The mOS for these AA/AOA patients was 56 weeks. Of interest to us at the time was that in the combined PR + SD group of $12 \mathrm{AA}$ and AOA patients, mOS was 119 weeks. Minimal activity was seen in 12 recurrent GBM patients; we found a median TTP of 8 weeks and mOS of 21 weeks. Lastly, we observed an interesting efficacy signal in that 
of five patients with brain stem tumors, three out of five were alive with SD of 77, 93 and 220 weeks at the time of publication. At the same time, these efficacy results reflect a total eflornithine daily dose of $6 \mathrm{~g} / \mathrm{m}^{2}$, which may have been too low for these patients.

Phase II study of eflornithine with $\alpha$-IFN for recurrent/progressive gliomas

A Phase II study of recombinant $\alpha$-IFN with eflornithine was conducted at the Mayo Clinic [22]. Patients in this study received eflornithine and $\alpha$-IFN subcutaneously (details in Table 1). No responses were observed in 29 patients with widely varying glioma grades: 4 with WHO grade 2, 5 with WHO grade 3, 13 with WHO grade 4 and 7 patients with unknown grades. SD that exceeded 6 months was reported in 24\% (7/29) of patients and was considered due to eflornithine administration. Grade 3 and 4 toxicities were mainly GI (nausea, vomiting, diarrhea), hematologic (leukopenia), myalgia, fever, chills and lethargy.

The daily eflornithine schedule used in the study was likely adequate based on the literature, but the duration of treatment was substantially lower than other studies conducted in patients with CNS malignancies. Given the small study size and heterogeneous glioma tumor grades treated, we cannot conclude whether eflornithine in combination with $\alpha$-IFN is an effective treatment.

\section{Randomized Phase III studies of adjuvant postirradiation PCV versus eflornithine-PCV in glioblastoma \& AG \\ Study design}

Two parallel Phase III randomized multi-institutional studies were conducted with the cooperation of patients from the MDACC, the Community Cancer Oncology Program of the MDACC and University of California-San Francisco (UCSF), with MDACC serving as the central registration site $[7,8]$. The protocol number was T92-0114 (CTEP) and DM92-035 (MDACC). Patient MRI scans were collected and reviewed when needed for assessment of progression. Tumor pathology slides for all patients registered to the study were reviewed by the referee pathologist, Janet M Bruner, MD, MDACC, using criteria published by Burger and the referee pathologist [23,24].

Patients were stratified by histology into two strata: GBM and gliosarcoma tumors in one strata and AG (WHO grade 3) that included AA, anaplastic oligodendroglioma (AO) and AOA tumors in the other study strata. The two strata had different accrual goals and constituted two separate studies. For AG patients, the trial was powered to detect an mOS of 54.5 months for the eflornithine combined with PCV compared with 36.2 months for PCV based on a one-sided log rank test with $\alpha=0.05$ and a power of $80 \%$.

The target sample size in the original 1989 protocol for the AG patient group was 303 patients, but because of slow accrual, the statistics were adjusted such that maximum accrual was set at 249 patients, a maximum accrual period of 7.7 years and a maximum study duration of 10.4 years.

The eflornithine and PCV treatment arm used the following starting dose and schedule: eflornithine, $3 \mathrm{~g} / \mathrm{m}^{2}$ orally every $8 \mathrm{~h}$ on days $1-14$; lomustine, $110 \mathrm{mg} / \mathrm{m}^{2}$ orally on day 15 ; procarbazine, $60 \mathrm{mg} / \mathrm{m}^{2} /$ day orally on days 22-35; vincristine, $1.4 \mathrm{mg} / \mathrm{m}^{2}$ iv. (maximum $2 \mathrm{mg}$ ) on days 22 and 43 ; and eflornithine, $3 \mathrm{~g} / \mathrm{m}^{2}$ orally every $8 \mathrm{~h}$ on days 29-42. The cycle was repeated at 8 -week intervals, for a total of seven cycles.

The PCV treatment arm used the following starting dose and schedule: lomustine, $110 \mathrm{mg} / \mathrm{m}^{2}$ orally on day 1; procarbazine $60 \mathrm{mg} / \mathrm{m}^{2}$ orally on days $8-21$; and vincristine, $1.4 \mathrm{mg} / \mathrm{m}^{2}$ iv. (maximum $2 \mathrm{mg}$ ) on days 8 and 29 . The cycle was repeated at 6-week intervals, for a total of seven cycles.

\section{Study results \& analyses}

The Kaplan-Meier method [25] was used to estimate OS and PFS survival curves as well as the corresponding median times and their CIs. To test for differences between survival curves the log rank test [26] and the Gehan version of the Wilcoxon test were used [27]. In addition, the Cox proportional hazards regression analysis was used to estimate hazard ratios and corresponding 95\% CIs [28]. Further, kernel smoothing was used to estimate the hazard functions over time and a cubic spline smooth of the Grambsch-Therneau-Schoenfeld [29] was used to estimate the $\log$ hazard ratio function over time. Since the treatment effect was clearly transient, hazard ratios for $0.5-5.0$ years were estimated in increments.

The GBM study accrued 272 evaluable patients and the AG study accrued 228 evaluable patients. The demographics of the AG study are summarized in Supplementary Table 1. Even though the studies did not accrue after June 1999, the studies were kept open to allow for follow-up until 2012 and, therefore, allowed opportunities to 


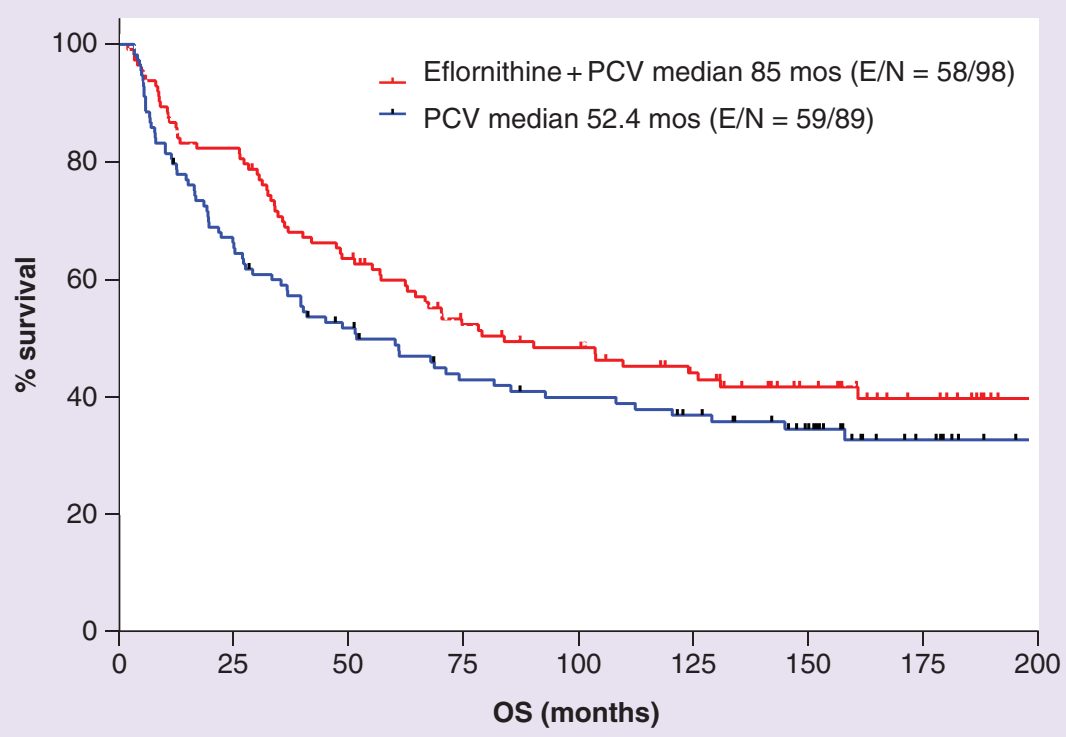

Figure 3. The year 2012 analysis for DM92-035 study for overall survival of patients with the diagnosis of anaplastic astrocytoma or anaplastic oligoastrocytoma. It was assumed, based on current molecular-based pathology definitions [15], that most anaplastic oligoastrocytoma would be considered anaplastic oligoastrocytoma today. The log rank comparison found hazard ratio $=0.75(95 \% \mathrm{Cl}: 0.52-1.07)$ with $\mathrm{p}=0.12$, the Gehan-Breslow-Wilcoxon test found $p=0.07$.

update OS in 2009 and 2012. While these data did not change the outcome and conclusions for GBM patients, it did improve the statistical reliability of OS for the AG study.

The initial publication of the multi-institutional post-RT Phase III study of PCV versus PCV and eflornithine for patients with GBM found no mOS improvement for patients treated on the PCV and eflornithine arm; in addition, mOS measured from time of tumor diagnosis at first surgery, was 13.3 and 14.2 months [7]. Supplementary Figure 1 shows results from the 2012 analysis demonstrating that mOS for the post-RT chemotherapy trial was 0.9 years ( $\sim 11$ months) for both arms of the study. Of interest is the observation that the pooled data for the 272 patients in the two arms demonstrated at 5 and 15 years after post-RT adjuvant chemotherapy started, that 8 and $4 \%$ of patients were still alive.

For study patients with the diagnosis of AG, the 2003 publication showed a substantial increase in mPFS (71.1 vs 37.5 months) and mOS (75.8 vs 61.1 months) for the eflornithine-PCV arm over PCV [8]. Figure 3 shows the Kaplan-Meier curve for OS from the 2012 database [30]. The mOS still favors the eflornithine-PCV arm and is now higher for both ( 85 vs 52.4 months), an improvement of 32.6 months based upon the addition of eflornithine to PCV. Table 2 summarizes the hazard ratio, $\mathrm{mOS}$ and $\mathrm{mPFS}$ for the 2002 database used in the 2003 paper [8] as well as from the 2009 and 2012 analyses [30].

To better understand why Kaplan-Meier survival curves for the eflornithine-PCV versus PCV arms appeared consistently different over time, but were not statistically significant by the global likelihood ratio (log rank) test, the data were analyzed using hazard ratio functions to compare eflornithine-PCV to PCV alone [8]. The premise was that a computed hazard ratio, that is the hazard rate for patients with PCV-alone treatment, divided by the hazard rate for patients with eflornithine-PCV, should be 1 if the hazard rates for the two arms are equivalent and there is no treatment effect. If, however, the hazard ratio is between 0 and 1 , it would indicate that the hazard rate is lower in patients with eflornithine-PCV treatment and that it is a more effective therapy than PCV.

Since the observations made in the original publication for PFS and OS showed that the hazard ratio for PFS and OS was not constant over time, we used hazard ratio changes over time to analyze the 2012 OS data and found it followed a similar pattern. Thus, the global analysis that assumes that the hazard function is constant over time is clearly invalid for OS data of both the 2002 and 2012 analyses. As a result, the hazard rates were evaluated to better understand the rate at which death or disease progression occurred in patients in each treatment arm over time. 


\begin{tabular}{|c|c|c|c|c|c|c|c|c|c|}
\hline Year & Path & $\begin{array}{l}\text { Eflornithine- } \\
\text { PCV mos, } \\
\text { months ( } 95 \% \\
\text { Cl) }\end{array}$ & $\begin{array}{l}\text { PCV mOS, } \\
\text { months ( } 95 \% \\
\text { Cl) }\end{array}$ & $\begin{array}{l}\text { Eflornithine- } \\
\text { PCV benefit, } \\
\text { months }\end{array}$ & $\begin{array}{l}\text { OS hazard } \\
\text { ratio }(95 \% \mathrm{Cl})\end{array}$ & $\begin{array}{l}\text { Log-rank } \\
\text { p-value }\end{array}$ & $\begin{array}{l}\text { Gehan- } \\
\text { Breslow- } \\
\text { Wilcoxon test } \\
\text { p-value }\end{array}$ & $\begin{array}{l}\text { PFS hazard } \\
\text { ratio }(95 \% \mathrm{Cl})\end{array}$ & $\begin{array}{l}\text { Log-rank } \\
\text { p-value }\end{array}$ \\
\hline $2002^{\dagger}$ & AG & 79 & 64 & 15 & $0.7(0.5-1.1)$ & 0.13 & 0.03 & $0.7(0.5-1.0)$ & 0.07 \\
\hline $2002^{\dagger}$ & AA & 71 & 46 & 25 & $0.7(0.5-1.0)$ & 0.08 & 0.04 & $0.7(0.5-1.4)$ & 0.08 \\
\hline 2009 & $\mathrm{AA} / \mathrm{AOA}$ & $85(57,132)$ & $52(28,85)$ & 33 & $0.7(0.5-1.1)$ & 0.11 & 0.07 & - & - \\
\hline 2012 & AG & $85(60,150)$ & $61(34,90)$ & 24 & $0.8(0.5-1.1)$ & 0.10 & 0.05 & - & - \\
\hline 2012 & AA & $76(55,130)$ & $42(25,85)$ & 34 & $0.7(0.5-1.1)$ & 0.09 & 0.05 & - & - \\
\hline 2012 & AA/AOA & $85(60,150)$ & $52(30,88)$ & 33 & $0.8(0.5-1.1)$ & 0.12 & 0.07 & - & - \\
\hline \multicolumn{10}{|c|}{$\begin{array}{l}\text { The method of Brookmeyer-Crowley was used to estimate the } 95 \% \mathrm{Cl} \text { for the median values in the table [31]. } \\
\text { 'From publication [8]. } \\
\text { AA: Anaplastic astrocytoma; AG: Anaplastic glioma; AOA: Anaplastic oligoastrocytoma; mOS: Median overall survival; OS: overall survival; PCV: Procarbazine, lomustine, vincristine; PFS } \\
\text { Progression-free survival. }\end{array}$} \\
\hline
\end{tabular}

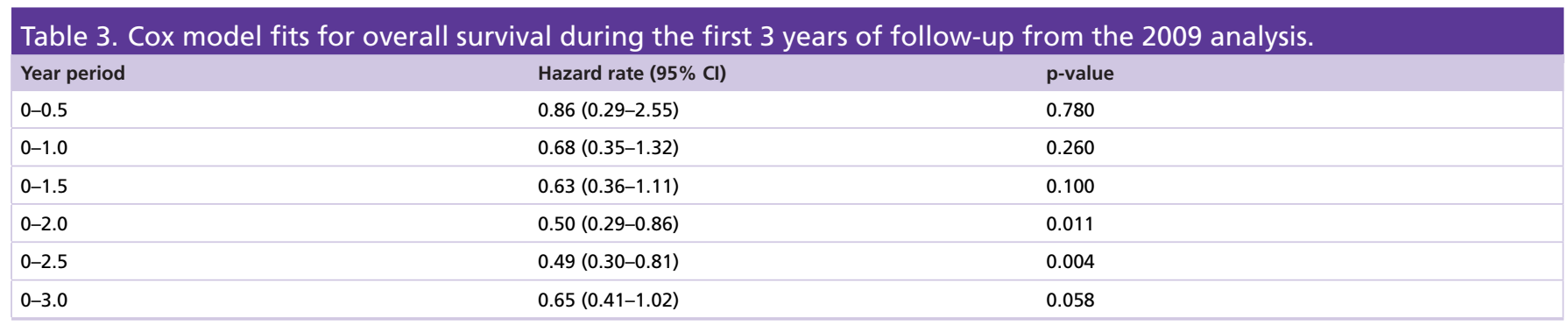

Recall that these hazard rates represent sensitive measures of the instantaneous event rate among the event-free cohorts (e.g., instantaneous death rates among the surviving patients).

To assess whether eflornithine-PCV and PCV alone patients have different event time distributions, a comparison of their respective hazard functions by estimating the ratios of the hazard functions was performed [32]. Hazard functions were estimated from the data using a fixed bandwidth kernel approach incorporating boundary kernels [33]. Since the magnitude of the hazard ratio indicates the difference in death rates, how the ratio changed over time to was assessed to see if the relative death rate between the groups was changing over time [29].

In the original paper, hazard functions converged at around 42 months for OS and at around 24 months for PFS [8]. For evaluable patients, when the 24-month follow-up period was partitioned and all observations beyond 24 months censored [29], the overall average crude hazard ratio in the first 24 months was 0.53 with $\mathrm{p}=0.020$ ( 0.55 with $\mathrm{p}=0.033$ after adjustment). When AA patients were analyzed separately, these relationships persisted $(\mathrm{p}=0.021)$. For analysis corresponding to the second period, all patients dying or censored during the first 24 months were excluded (leaving 166 patients for analysis). After 24 months, the average hazard ratio was 1.07 with $\mathrm{p}=0.83$. A similar relationship was seen for PFS, where the adjusted hazard ratio for AG patients was 0.63 with $\mathrm{p}=0.033$; for the AA subgroup; however, the hazard ratio was 0.67 with $\mathrm{p}=0.099$. These findings implied that the survival advantage for $\mathrm{AG}$ patients with no more than 12 months of treatment with eflornithine-PCV was limited to the first 2 years after treatment compared with patients treated with PCV alone.

This statistical analysis for OS for this study was also performed in 2009. The OS hazard rate for 114 eflornithinePCV and 114 PCV patients is shown for eflornithine-PCV (solid line) and PCV (dashed line) is in Figure 4A. Figure $4 \mathrm{~B}$ shows the log hazard ratio and $95 \%$ CIs. The Cox model fit between 0 and 2.5 years had a hazard ratio of 0.49 and p-value $=0.004$ (see Table 3 ).

While the patient database of 2003 was updated in 2009 and again in 2012, only OS data are presented in detail here. PFS determination for AG tumors is less reliable 7 and 10 years after the 2002 analysis, whereas OS data became more precise with more events over time and are less ambiguous. Over the 10-year period the hazard ratios for AG, AA and AA and AOA combined remain relatively constant (Table 2). The differential in mOS for AG throughout the 10-year period continues to favor the eflornithine-PCV arm with a difference of 15 months that 
(A)

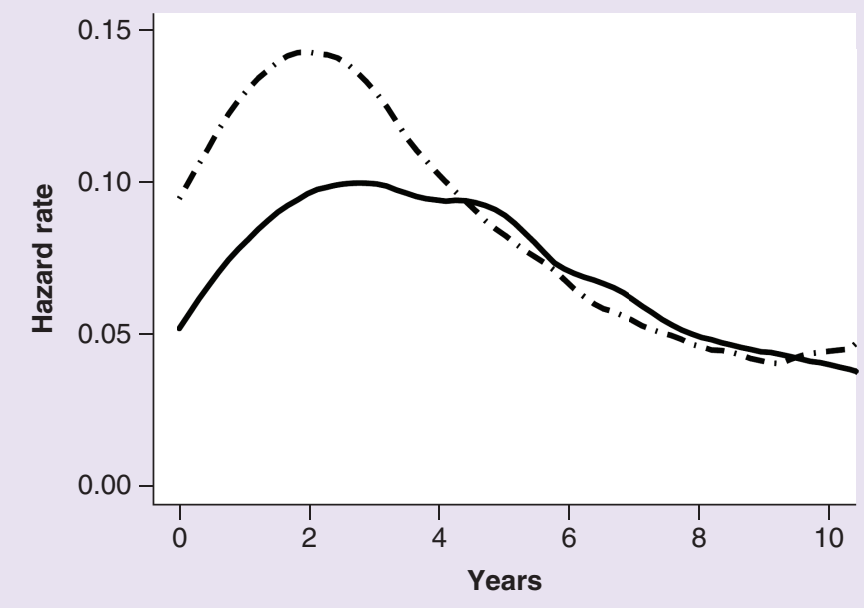

(B)

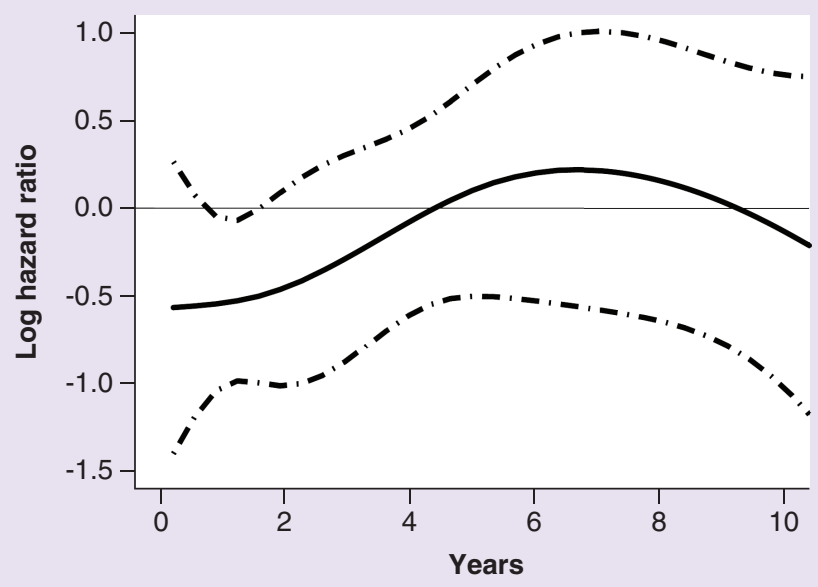

Figure 4. Hazard rate and hazard ratio plots for the reanalyzed 2009 anaplastic glioma dataset. ( A) Overall survival hazard rate for 114 patients receiving eflornithine-PCV (solid line) and 114 patients receiving procarbazine, lomustine, vincristine (dashed line). (B) Shows the log hazard ratio (solid line) and $95 \% \mathrm{Cl}$ (dashed line). The Cox model fit between 0 and 2.5 years had a hazard ratio $=0.49$ and $p=0.004$ (see Table 2).

increases to 24 months by 2009. Similarly, for AA the differential is 25 months in 2002 and 34 months in 2012. Since most AOA tumors today are called AA based on their molecular-genetic profiles, it was decided to combine AA and AOA tumors into one group. Figure 3 shows the Kaplan-Meier plot for the combined AA and AOA patients and the mOS difference of 33 months. Interestingly, the difference in mOS for this group is 33 months at both years 2009 and 2012 (Table 2).

The AO histology, while distinct among AG with respect to response to RT and alkylating agents, a total number of $\mathrm{AO}$ in the eflornithine-PCV (DM92-035) protocol were insufficient for an in-depth statistical analysis [8]. Nonetheless, the activity signal seen for $\mathrm{AO}$ with eflornithine (Supplementary Figure 2) is consistent with what was observed for the AA and AOA tumors. What may be important to this observation is that all the AO in this protocol were classified without consideration for chromosome $1 \mathrm{p} / 19 \mathrm{q}$ co-deletion. It is reasonable to assume that the presence of chromosome $1 \mathrm{p}$ and $19 \mathrm{q}$ co-deletion will be randomly distributed between the two study arms; however, it would be instructive to determine if the absence of chromosome $1 \mathrm{p} / 19 \mathrm{q}$ co-deletion impacts eflornithine efficacy.

\section{Toxicity}

The safety profile for eflornithine when dosed in combination with PCV, is shown for the 500 patients in the GBM and AG studies, is summarized in Table 4. For toxicity grades 1 and 2, diarrhea is prominent (25.4 and 17.4\%) as is anemia (12.5 and 15.6\%). In addition, grade 2 thrombocytopenia was observed in $10.5 \%$. Interestingly, few grades 3 and $4 \mathrm{AE}$ were observed and none reached 10\%. Grade 3 diarrhea was seen in $6 \%$ but almost none at grade $4(<1 \%)$. Grade 3 and 4 myelosuppression appeared little affected by the addition of eflornithine to PCV. Thus, the addition of eflornithine to cytotoxic PCV appears well tolerated by patients.

\section{Discussion}

Eflornithine is an active agent against some solid tumors and leukemia [1], and in the prevention of malignant colon polyps [34]; however, it is not currently FDA approved for use in the treatment of cancer. Analyses of more recent data in this report support and extend earlier reports that demonstrate the likely benefit of eflornithine alone and combined with nitrosourea-based therapies to treat malignant gliomas at appropriate daily doses and study durations.

In earlier publications, eflornithine activity was described in the treatment of recurrent $\mathrm{AA}, \mathrm{AOA}$ and $\mathrm{AO}$ tumors in patients [9]. In combination with PCV, eflornithine showed an increase in OS compared with PCV alone in a randomized Phase III postirradiation adjuvant clinical trial in patients with AGs [8]. In that original report, it was 


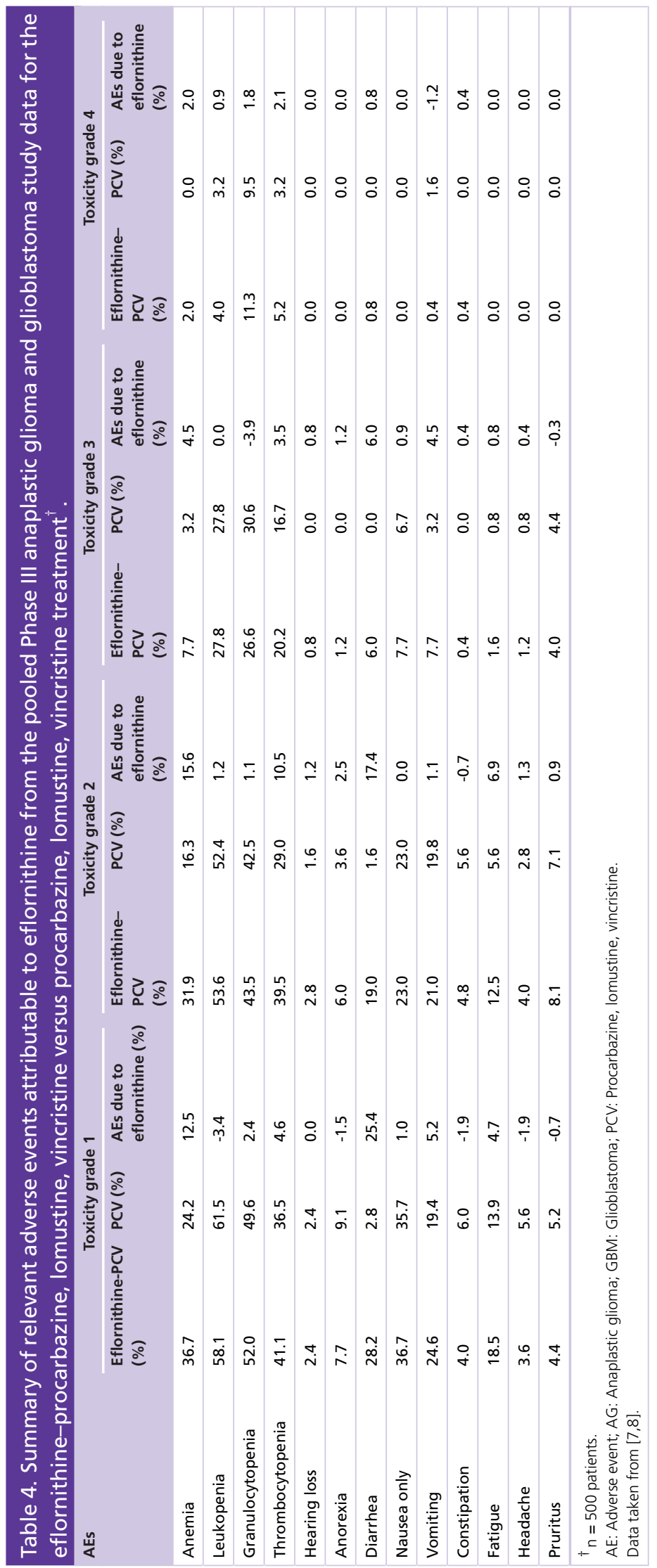


not possible to illustrate a statistically significant difference in mOS by the log-rank test as there were too few events to show statistical significance at the time of publication. However, in that Phase III study, statistical improvement in PFS based on hazard rate was illustrated.

Utilizing data from 2009 and 2012 [30], that were 7 and 10 years, respectively, after the analysis of the Phase III report [8], we found that only $57 \%$ of eflornithine-PCV patients and $63 \%$ of PCV patients had died. Comparison of OS differences between the two arms by log rank found p-values of 0.09-0.12 depending on histology grouping (Table 2) while comparison of OS by the Gehan modification of the Wilcoxon analysis found statistically significant differences in favoring eflornithine-PCV with p-values of 0.05-0.07. Furthermore, interpretation of the Cox model hazard ratio for OS found that the eflornithine-PCV combination produced its differential benefit in the first 2.5 years of study with $\mathrm{p}=0.04$. The same analysis, conducted for PFS in the original publication, showed the benefit of eflornithine-PCV treatment peaked a bit earlier at 0.5 years and persisted for about 2 years [8]. The studies described in this review support the conclusion that appropriate dose levels in a defined regimen of eflornithine have a tangible clinical effect on PFS and OS for AG tumors.

It is important to mention that, aside from the Chamberlain study [17], none of the AA and AO studies reported in this review utilized molecular profiling to define histology, malignancy grade and extent of benefit expected from RT and alkylating agent therapy. While it is highly unlikely that AO patients were mistakenly placed in the AA category based on the criteria used by the referee neuropathologists at MDACC and UCSF at the times of these studies [23], it is likely that those patients today considered to be genetically GBM were called AA, since the neuropathologists at these institutions, for consistency in classification, required pseudopalisading necrosis for the diagnosis of GBM. Thus, it is likely that many (10-15\%) of the AA patients in the Phases II and III at progression behaved as GBM.

\section{Conclusion}

Eflornithine has shown therapeutic activity for patients with various forms of cancer including infiltrating gliomas. It showed clinically meaningful increase in median overall survival in patients with recurrent anaplastic gliomas when used alone and in combination with PCV. Eflornithine toxicity is relatively mild in comparison to currently used chemotherapies for gliomas with reversible hearing impairment and tinnitus being the most noticeable to patients.

\section{Future perspective}

To provide the basis for seeking regulatory approval of eflornithine for the treatment of anaplastic astrocytomas, a multicenter randomized Phase III trial (the "STELLAR" trial; NCT02796261) is currently active in the USA, Belgium, Canada, Germany, Italy, and The Netherlands. The objective of the STELLAR trial is to provide the necessary evidence to support an application for market approval at study conclusion. Based upon the results described above in Phase II and Phase III studies with eflornithine, the STELLAR trial was designed to compare the efficacy and safety of oral eflornithine in combination with oral lomustine, compared to oral lomustine taken alone, in treating patients with AA that has recurred or progressed after radiation therapy and temozolomide (Temodar $\AA$ ) chemotherapy. The primary outcome measure is overall survival with secondary measures including progression-free survival and overall response rate. Based upon its putative mechanism of action, clinical history and potential to be used alone and in combination with other agents, it is conceivable that eflornithine will, in future years, be found useful alone and in drug combination therapies for other cancers in adults and children.

\section{Supplementary data}

To view the supplementary data that accompany this paper please visit the journal website at: www.futuremedicine.com/doi/full/10.2217/cns-2017-0031

\section{Open access}

This work is licensed under the Attribution-NonCommercial-NoDerivatives 4.0 Unported License. To view a copy of this license, visit http://creativecommons.org/licenses/by-nc-nd/4.0/ 
Financial \& competing interests disclosure

Studies previously published by the lead author (VA Levin) were previously funded by the NIH CA13525, CA55261 and US FDA FD-R-000492-02 and NIH U01CA45809 (CCOP). VA Levin is a paid consultant for Orbus Therapeutics. The authors have no other relevant affiliations or financial involvement with any organization or entity with a financial interest in or financial conflict with the subject matter or materials discussed in the manuscript apart from those disclosed.

No writing assistance was utilized in the production of this manuscript.

Ethical conduct of research

The authors state that they have obtained appropriate institutional review board approval or have followed the principles outlined in the Declaration of Helsinki for all human or animal experimental investigations. In addition, for investigations involving human subjects, informed consent has been obtained from the participants involved.

\section{References}

1. Shantz LM, Levin VA. Regulation of ornithine decarboxylase during oncogenic transformation: mechanisms and therapeutic potential. Amino Acids 33(2), 213-223 (2007).

2. Bachmann AS, Levin VA. Clinical applications of polyamine-based therapeutics. In: Polyamine Drug Discovery. Woster PM, Casero R (Eds). RSC Publishing, London, UK, 257-276 (2012).

3. Clinical Investigator Brochure Ornidyl (eflornithine hydrochloride). Report No.: X-81-02. Merrell Dow Research Institute, Cincinnati, USA (1987).

4. Carbone PP, Douglas JA, Thomas J et al. Bioavailability study of oral liquid and tablet forms of $\alpha$-difluoromethylornithine. Clin. Cancer Res. 6(10), 3850-3854 (2000).

5. Levin VA, Chamberlain MC, Prados MD et al. Phase I-II study of eflornithine and mitoguazone combined in the treatment of recurrent primary brain tumors. Cancer Treat. Rep. 71(5), 459-464 (1987).

6. Prados M, Rodriguez L, Chamberlain M, Silver P, Levin V. Treatment of recurrent gliomas with 1,3-bis(2-chloroethyl)-1-nitrosourea and $\alpha$-difluoromethylornithine. Neurosurgery 24(6), 806-809 (1989).

7. Levin VA, Uhm JH, Jaeckle KA et al. Phase III randomized study of postradiotherapy chemotherapy with $\alpha$-difluoromethylornithine-procarbazine, $\mathrm{N}$-(2-chloroethyl)- $\mathrm{N}^{\prime}$-cyclohexyl-N-nitrosurea, vincristine (DFMO-PCV) versus PCV for glioblastoma multiforme. Clin. Cancer Res. 6(10), 3878-3884 (2000).

8. Levin VA, Hess KR, Choucair A et al. Phase III randomized study of postradiotherapy chemotherapy with combination a-difluoromethylornithine-PCV versus PCV for anaplastic gliomas. Clin. Cancer Res. 9(3), 981-990 (2003).

9. Levin VA, Prados MD, Yung WK, Gleason MJ, Ictech S, Malec M. Treatment of recurrent gliomas with eflornithine. J. Natl Cancer Inst. 84(18), 1432-1437 (1992).

10. Griffin CA, Slavik M, Chien SC et al. Phase I trial and pharmacokinetic study of intravenous and oral $\alpha$-difluoromethylornithine. Invest. New Drugs 5(2), 177-186 (1987).

11. Abeloff MD, Slavik M, Luk GD et al. Phase I trial and pharmacokinetic studies of $\alpha$-difluoromethylornithine - an inhibitor of polyamine biosynthesis. J. Clin. Oncol. 2(2), 124-130 (1984).

12. NIH Clinical Trials.gov Identifier: NCT02796261. https://clinicaltrials.gov/ct2/show/NCT02796261

13. Warrell RP, Coonley CJ, Burchenal JH. Sequential inhibition of polyamine synthesis. Cancer Chemother. Pharmacol. 11(2), 134-136 (1983).

14. Levin VA, Crafts DC, Norman DM, Hoffer PB, Spire JP, Wilson CB. Criteria for evaluating patients undergoing chemotherapy for malignant brain tumors. J. Neurosurg. 47(3), 329-335 (1977).

15. Louis DN, Perry A, Reifenberger G et al. The 2016 World Health Organization classification of tumors of the central nervous system: a summary. Acta Neuropathol. 131(6), 803-820 (2016).

16. Wong ET, Hess KR, Gleason MJ et al. Outcomes and prognostic factors in recurrent glioma patients enrolled onto Phase II clinical trials. J. Clin. Oncol. 17(8), 2572-2578 (1999).

17. Chamberlain MC. Salvage therapy with lomustine for temozolomide refractory recurrent anaplastic astrocytoma: a retrospective study. $J$. Neurooncol. 122(2), 329-338 (2015).

18. Hung DT, Deen DF, Seidenfeld J, Marton LJ. Sensitization of 9L rat brain gliosarcoma cells to 1,3-bis(2- chloroethyl)-1-nitrosourea by $\alpha$-difluoromethylornithine, an ornithine decarboxylase inhibitor. Cancer Res. 41(7), 2783-2785 (1981).

19. Marton LJ, Levin VA, Hervatin SJ, Koch-Weser J, McCann PP, Sjoerdsma A. Potentiation of the antitumor therapeutic effects of 1,3-bis(2-chloroethyl)-1-nitrosourea by $\alpha$-difluoromethylornithine, an ornithine decarboxylase inhibitor. Cancer Res. 41(11 Pt 1), 4426-4431 (1981).

20. Bjerkvig R, Oredsson SM, Marton LJ, Linden M, Deen DF. Cell cycle age response of 9L cells to 1,3-bis(2-chloroethyl)-1-nitrosourea and modification by $\alpha$-difluoromethylornithine. Cancer Res. 43(4), 1497-1500 (1983). 
21. Oredsson SM, Deen DF, Marton LJ. Influence of polyamine depletion caused by $\alpha$-difluoromethylornithine, an enzyme-activated irreversible inhibitor of ornithine decarboxylase, on alkylation- and carbamoylation-induced cytotoxicity in 9L rat brain tumor cells in vitro. Cancer Res. 43(10), 4606-4609 (1983).

22. Buckner JC, Burch PA, Cascino TL, O’Fallon JR, Scheithauer BW. Phase II trial of recombinant interferon- $\alpha-2 a$ and eflornithine in patients with recurrent glioma. J. Neurooncol. 36(1), 65-70 (1998).

23. Burger PC, Scheithauer BW, Vogel FS. Surgical Pathology of the Nervous System and its Coverings (3rd Edition). Churchill Livingstone, NY, USA, 214-234 (1991).

24. Berger MS, Leibel SA, Bruner JM, Finlay JL, Levin VA. Primary cerebral tumors. In: Cancer in the Nervous System (2nd Edition). Levin VA (Ed.). Oxford University Press, Oxford, UK, 75-148 (2002).

25. Kaplan EL, Meier P. Nonparametric estimates from incomplete observations. J. Am. Stat. Assoc. 53, 457-481 (1958).

26. Mantel N. Evaluation of survival data and two new rank order statistics arising in its consideration. Cancer Chemother. Rep. 50(3), 163-170 (1966).

27. Gehan EA. A generalized two-sample Wilcoxon test for doubly censored data. Biometrika 52(3), 650-653 (1965).

28. Cox DR. Regression models and life tables. J. R. Stat. Soc. Series B Stat. Methodol. 34(2), 187-220 (1972).

29. Hess KR. Graphical methods for assessing violations of the proportional hazards assumption in Cox regression. Stat. Med. 14(15), $1707-1723$ (1995).

30. Levin VA, Hess KR, Choucair AK et al. Final report for evaluable patients treated on DM92-035, Phase III randomized study of post-irradiation PCV versus DFMO-PCV, for anaplastic gliomas (AG). Neuro Oncol. 14(Suppl. 6), vi74 (2012).

31. Brookmeyer R, Crowley J. A confidence interval for the median survival time. Biometrics 38(1), 29-41 (1982).

32. Hess KR, Levin VA. Getting more out of survival data by using the hazard function. Clin. Cancer Res. 20(6), 1404-1409 (2014).

33. Hess KR, Serachitopol DM, Brown BW. Hazard function estimators: a simulation study. Stat. Med. 18(22), 3075-3088 (1999).

34. Meyskens FL Jr, McLaren CE, Pelot D et al. Difluoromethylornithine plus sulindac for the prevention of sporadic colorectal adenomas: a randomized placebo-controlled, double-blind trial. Cancer Prev. Res. 1(1), 32-38 (2008). 
